\title{
DNMT3a mutations in high-risk myelodysplastic syndrome parallel those found in acute myeloid leukemia
}

Blood Cancer Journal (2011) 1, e9; doi:10.1038/bcj.2011.7; published online 11 March 2011

Recent work has identified heterozygous mutations in DNMT3a in $4.1-22.1 \%$ of patients with acute myeloid leukemia (AML). ${ }^{1,2}$ Here we show that $3.3 \%$ of patients with high risk myelodysplastic syndrome (MDS) also possess heterozygous mutations in the methyltransferase domain of DNMT3a. Our results suggest that DNMT3a mutations may precede the development of AML.

MDS is a heterogenous group of clonal bone marrow disorders characterized by ineffective hematopoiesis, cytopenias and increased risk of AML. On a cellular level, there is an increased rate of proliferation accompanied by an increase in apoptosis. Currently approved treatments include supportive therapy with transfusion and growth factors followed by disease modifying agents lenalidomide, and the hypomethylating agents, azacitidine and decitabine. ${ }^{3}$ Azacitidine and decitabine are often reserved for treatment of patients with high-risk disease due to their potential toxicities and limited efficacy for lower risk patients. ${ }^{3,4}$ These agents are thought to act by altering aberrant methylation patterns seen in patients with MDS through inhibition of the DNA methyltransferases (DNMTs). ${ }^{4}$

DNMTs comprise a family of methyltransferase proteins that catalyze the methylation of cytosine within CpG dinucleotides. These CpG dinucleotides are frequently found within the upstream regulatory region of genes and their methylation can lead to gene silencing. ${ }^{5}$ Studies of DNA methylation in cancer and MDS have shown altered methylation patterns in these diseases particularly with hypermethylation of tumor suppressors and hypomethylation of proto-oncogenes. ${ }^{6-8}$ These epigenetic changes are possibly due to alteration in the activity or target specificity of DNMTs.

Recent studies in AML have found mutations in the DNA methyltransferase DNMT3a. ${ }^{1,2}$ Yamashita et al. reported that $3 / 74(4.1 \%)$ of AML patients in their series harbored heterozyogous DNMT3a mutations at the R882 position. ${ }^{1}$ More recently, Ley et al. identified a number of heterozygous DNMT3a mutations in 62/281 (22.1\%) of AML patients in their series. Although the majority of these mutations affected the R882 position, additional mutations were identified. The authors attribute the discrepancy in mutation frequency to the use of massively parallel sequencing. Despite the increased frequency of mutation, the R882 mutations comprised the majority of DNMT3a mutations and were associated with a significantly worse prognosis. $^{2}$

Following the report by Yamashita et al. of heterozygous mutations within the methyltransferase domain of DNMT3a in patients with AML, ${ }^{1}$ we wondered whether MDS samples also bear such mutations. If so, such mutations would precede the development of leukemia and may be important in the pathogenesis of MDS. We therefore focused on patients with high risk MDS - including refractory anemia with excess blasts I or II (RAEB-I, RAEBII) - both of which carry a high likelihood of transforming to $\mathrm{AML}$, but do not possess the pathological features of AML.
The MT domain of DNMT3a contains eight exons. Initially, we amplified and sequenced all eight exons from 62 RAEB-I and RAEBII patients using genomic DNA extracted from bone marrow samples. We identified six heterozygous mutations in five patients from this sample (Table 1). Two were silent base pair changes and were not examined further. Two were guanine to adenosine substitutions resulting in the substitution of histidine for arginine at amino acid $882(\mathrm{R} 882 \mathrm{H})$. One was a cytosine to thymine substitution resulting in the substitution of cysteine for arginine at amino acid 882 (R882C) and the last mutation was a guanine to adenosine substitution resulting in the substitution of tyrosine for cysteine at amino acid 709 (C709Y). Given that these missense mutations were found exclusively in the third and seventh amplicons, we amplified only these segments in an additional set of 38 RAEB-I and RAEB-II patients and identified one additional heterozygous R882C mutation. In total we identified four patients who possess R882 mutations identical to those previously described in AML and one of these four also harbored a C709Y mutation.

We next determined whether these mutations were somatically derived within the dysplastic marrow of these patients by examining DNA derived from buccal swabs of the patients. In principle, buccal DNA should contain the germline sequence, although saliva is known to be contaminated with hematopoietic cells (particularly leukocytes).

We amplified and sequenced the region of R882 and C709. The R882 mutation was clearly somatic in one of the patients examined (Figure 1, patient 55). The buccal DNA of patient 37 showed a low ratio of the mutated sequence. We hypothesized that this low-abundance mutant sequence may be due to contamination with mutation-bearing hematopoietic cells. To test this, we repeated the amplification and sequencing on a second buccal swab from patient 37 and found that the mutation was absent, indicating previous sample contamination and that the mutation arose somatically within the marrow (Figure 1, patient 37).

A third patient (68) also possessed the mutant allele in the buccal swab, but the low ratio of wild type to mutant in the

Table 1 Analysis of bone marrow and buccal DNA showing somatic missense mutations in patients 37,55 , and 68 and silent mutations in patients 14 and 33

\begin{tabular}{llll}
\hline Patient & Diagnosis & Mutation & Somatic \\
\hline 14 & RAEB I & Ser892Ser $(\mathrm{A}>\mathrm{T})$ & Not examined \\
33 & RAEB I & Asn717Asn $(\mathrm{C}>\mathrm{T})$ & Not examined \\
37 & RAEB II & Cys709Tyr $(\mathrm{G}>\mathrm{A})$ & Somatic $^{\mathrm{a}}$ \\
& & Arg882Cys $(\mathrm{C}>\mathrm{T})$ & \\
55 & RAEB I & Arg882His $(\mathrm{G}>\mathrm{A})$ & Somatic \\
68 & RAEB II & Arg882Cys $(\mathrm{C}>\mathrm{T})$ & Likely $^{\mathrm{b}}$ \\
\hline
\end{tabular}

anitial buccal swab shows decreased frequency of mutation from bone marrow, repeat buccal swab shows clearly that the mutation arose somatically.

bThere is a decreased frequency of mutation in the buccal swab compared with the bone marrow but we hypothesize that this reflects contamination. 


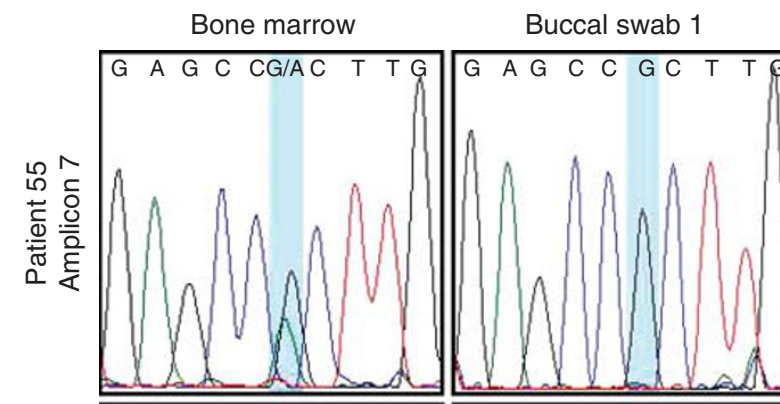

Buccal swab 2

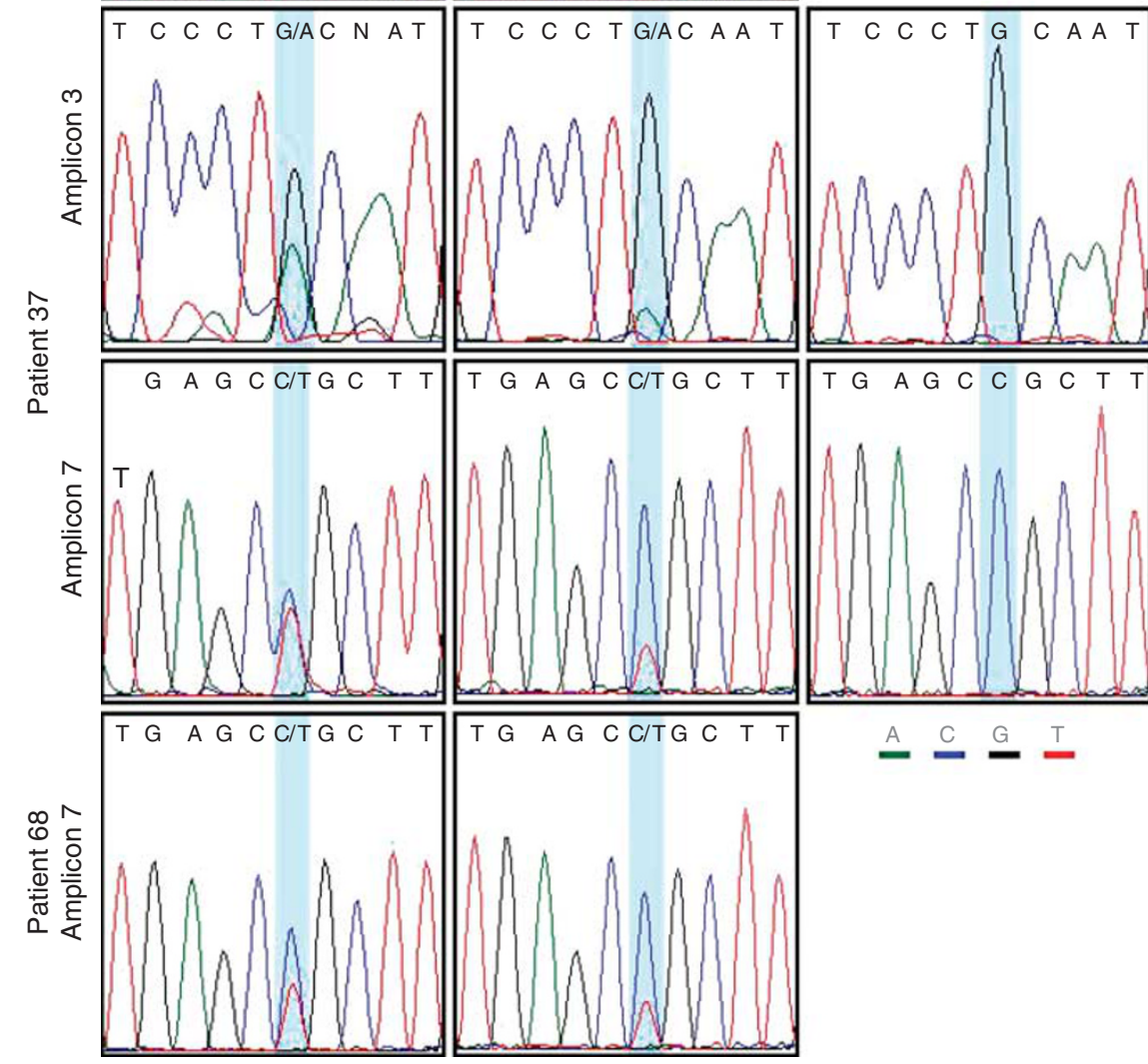

Figure 1 Sequencing results for patient 37, 52 and 68. Patient 52 harbors a heterozygous somatic mutation in amplicon 7 in bone marrow specimens. Patient 37 harbors heterozygous somatic mutations in amplicons 3 and 7 in bone marrow with reduced frequency in the first buccal swab and no mutation in the second buccal swab. Patient 68 harbors a heterozygous somatic mutation in amplicon 7 in bone marrow specimens with reduced frequency in the buccal swab.

sample suggests that the R882 H mutation was somatic (Figure 1, patient 68).

A final patient (52) also possessed a $\mathrm{R} 882 \mathrm{H}$ mutation, but this could not be proved to be somatic because of contamination in the buccal swab and was thus inconclusive.

The contamination of the buccal swab raises an important discussion point. Of note Yamashita et al. used T cells and Ley et al. used skin biopsies as a source of germline DNA for their patients and reported that all of the DNMT3a mutations were somatically acquired within the bone marrow. ${ }^{1,2}$ Our varied results using different buccal samples for patient 37 and the altered frequency of mutation in patient 68 suggest that buccal swabs may not be a pure population of non-hematopoietic cells and that multiple swabs should be analyzed with clearly defined sequence ratios before deciding whether a mutation is germline.

The mutations we describe here are identical to those previously described in AML. Yamashita et al. suggested that $\mathrm{R} 882 \mathrm{H}$ mutations decreased the activity of DNMT3a and Ley et al. reported that there were 182 genomic regions with significantly altered methylation in R882 $\mathrm{H}$ mutants as compared with matched AML genomes from patient's with wild-type DNMT3a. ${ }^{1}$ Ley et al. also found that R882 mutations were associated with a worse prognosis in AML patients. ${ }^{2}$ Taken together these data suggest that R882 mutations are associated with altered activity in DNMT3a. We now show that these alterations are present in MDS before development of frank leukemia. Future studies will help to illuminate the effect that these changes have on gene function, genomic methylation and overall expression patterns. The presence of these mutations before leukemic transformation may become a useful prognostic marker of likelihood of progression and of poor prognosis although additional study is needed to clarify this.

\section{Conflict of interest}

The authors declare no conflict of interest. 
M Ewalt ${ }^{1}$, NG Galili ${ }^{2,3}$, M Mumtaz $^{2}$, M Churchill $^{2}$, S Rivera ${ }^{2}$, F Borot $^{2}$, A Raza $^{2,3}$ and S Mukherjee ${ }^{2,3}$

${ }^{1}$ Department of Pathology, Columbia University Medical Center, New York, New York, USA;

${ }^{2}$ Herbert Irving Cancer Center, Columbia University Medical Center, New York, New York, USA and

${ }^{3}$ Department of Medicine, Division of Oncology, Columbia

University Medical Center, New York, New York, USA E-mail:sm3252@columbia.edu

\section{References}

1 Yamashita Y, Yuan J, Suetake I, Suzuki H, Ishikawa Y, Choi YL et al. Array-based genomic resequencing of human leukemia. Oncogene 2010; 29: 3723-3731.

2 Ley TJ, Ding L, Walter MJ, McLellan MD, Lamprecht T, Larson DE et al. DNMT3A mutations in acute myeloid leukemia. N Engl J Med 2010; 363: 2424-2433.

3 Galili N, Raza A. Pharmacotherapy of myelodysplastic syndromes. Expert Opin Pharmacother 2010; 11: 1889-1899.
4 Garcia-Manero G. Demethylating agents in myeloid malignancies. Curr Opin Oncol 2008; 20: 705-710.

5 Jones PA, Liang G. Rethinking how DNA methylation patterns are maintained. Nat Rev Genet 2009; 10: 805-811.

6 Xu F, Li X, Wu L, Zhang Q, Yang R, Yang Y et al. Overexpression of the EZH2, RING1 and BMI1 genes is common in myelodysplastic syndromes: relation to adverse epigenetic alteration and poor prognostic scoring. Ann Hematol 2010 (e-pub ahead of print 2 December 2010).

7 Mori N, Yoshinaga K, Tomita K, Ohwashi M, Kondoh T, Shimura H et al. Aberrant methylation of the RIZ1 gene in myelodysplastic syndrome and acute myeloid leukemia. Leuk Res 2010 (e-pub ahead of print 7 September 2010).

8 Jones PA, Baylin SB. The epigenomics of cancer. Cell 2007; 128: 683-692.

(c) This work is licensed under the Creative Commons SOWERाEHIS RESERVED Attribution-NonCommercial-Share Alike 3.0 Unported License. To view a copy of this license, visit http://creativecommons. org/licenses/by-nc-sa/3.0/ 